\title{
Dietary intervention to reduce meat intake by $50 \%$ in University students - a pilot study
}

\author{
T. Holloway, A. M. Salter and F. S. McCullough \\ Division of Nutritional Sciences, School of Biosciences, University of Nottingham, Sutton Bonington Campus, \\ Loughborough, UK
}

The ability to supply an increasing global demand for meat and the impact of farm animal production on climate change have recently come under scrutiny. A recent report from the UK Government Office for Science ${ }^{(1)}$ suggests that, "a reduction in the amount of meat consumed in high- and middle-income countries would have multiple benefits: a reduced demand for grain, leading to lower greenhouse gas emissions, and a positive effect on health".

It has not yet been established if directed education would enable healthy individuals to reduce meat intake, in terms of compliance and acceptability of meat substitutes and alternatives within the diet. The hypothesis of the present study was that with appropriate support, free living subjects can reduce their meat intake by up to $50 \%$ without significant effects on health and well-being and without adversely affecting lifestyle.

Twenty six healthy subjects were recruited from the student population of the University of Nottingham. At baseline they completed a 7-day diet diary and health and well-being questionnaire. Anthropometric measurements including BMI and total body fat, using the Bod-pod, were recorded. The intervention included two information-based motivational events related to meat reduction including an introduction to meat alternatives. Based on their reported meat intakes, subjects were provided with sufficient non-meat alternatives to replace approximately $50 \%$ of the energy previously obtained from meat. The non-meat alternatives included a range of commercially available, vegetable based protein-rich, products. Subject compliance was monitored weekly over the 4-week meat reduction period and the variety of non-meat alternatives varied according to personal preferences. Subjects performed another 7 day diet diary and questionnaire at the end of the trial and anthropometric measurements were repeated.

A total of 25 subjects (10 male/15 female) completed the trial. All subjects reported successfully incorporating non-meat alternatives into their diets. Average age of subjects was 21.2 y (SD 3.3 y) Baseline and final anthropometric data are reported below.

\begin{tabular}{llcrr}
\hline & \multicolumn{2}{c}{ Baseline } & \multicolumn{2}{c}{ After } \\
\cline { 2 - 5 } \cline { 4 - 4 } & Mean & SD & Mean & SD \\
\hline Weight $(\mathrm{kg})$ & 66.12 & 12.90 & $64.85^{*}$ & 11.8 \\
BMI $\left(\mathrm{kg} / \mathrm{m}^{2}\right)$ & 23.18 & 3.22 & $22.75^{*}$ & 2.96 \\
Body fat $(\%)$ & 24.25 & 6.51 & 24.08 & 6.49 \\
\hline Values are means and standard deviation of 25 subjects (paired student t-test), * $p<0.05$. &
\end{tabular}

There were small but significant decreases in weight $(p<0.01)$ and BMI $(p<0.001)$ but no change in body composition $($ as reflected in the percent body fat) suggesting that meat substitution resulted in a modest decrease in energy consumption. Analysis of diet diaries will provide data on the impact of meat replacement on macro- and micro-nutrient intake.

Overall the results indicate that, with appropriate support healthy, young individuals can successfully replace up to half of their meat intake with non-meat alternatives.

This work was partly supported by a grant from the University of Nottingham.

1. Foresight. The Future of Food and Farming (2011) Final Project Report. The Government Office for Science, London. 